\title{
Gluon GPDs and exclusive photoproduction of quarkonium in forward region
}

\author{
Z. L. Cui ${ }^{1,2, a}$, M. C. $\mathbf{H u}^{1,2}$, J. P. Ma $\mathbf{M}^{1,2,3}$ \\ ${ }^{1}$ Institute of Theoretical Physics, Chinese Academy of Sciences, P.O. Box 2735, Beijing 100190, China \\ ${ }^{2}$ School of Physical Sciences, University of Chinese Academy of Sciences, Beijing 100049, China \\ ${ }^{3}$ School of Physics and Center for High-Energy Physics, Peking University, Beijing 100871, China
}

Received: 3 December 2018 / Accepted: 13 September 2019 / Published online: 1 October 2019

(C) The Author(s) 2019

\begin{abstract}
Forward photoproduction of $J / \psi$ can be used to extract generalized parton distributions (GPDs) of gluons. We analyze the process at twist-3 level and study relevant classifications of twist-3 gluon GPDs. At leading power or twist- 2 level the produced $J / \psi$ is transversely polarized. We find that at twist- 3 the produced $J / \psi$ is longitudinally polarized. Our study shows that in the high-energy limit the twist3 amplitude is only suppressed by the inverse power of the heavy quark mass relatively to the twist- 2 amplitude. This indicates that the power correction to the cross-section of unpolarized $J / \psi$ can have a sizable effect.The poles in the hard factor convoluted with twist-3 gluon GPDs may concern the GPDs discontinuities, which will be briefly discussed in this work. We have also derived the amplitude of the production of $h_{c}$ at twist-3, but the result contains end-point singularities. The production of other quarkonia has been briefly discussed.
\end{abstract}

\section{Introduction}

Properties of hadrons are described by their matrix elements of QCD operators. These matrix elements are nonperturbative. They can be calculated only with nonperturbative methods, or extracted from experimental data. A class of such matrix elements are Generalized Parton Distributions(GPDs) introduced in [1-3]. In [4,5] it has been shown that one can obtain the quark or gluon contributions to the proton spin from quark or gluon twist-2 GPDs, respectively. Therefore, studies of GPDs and relevant processes both in theory and experiment will help to solve the so-called proton spin crisis and provide more information about the hadron's inner structure.

\footnotetext{
a e-mail: cuizelun@itp.ac.cn
}

There is a class of exclusive processes which can be used to extract GPDs. They can be extracted from deeply virtual Compton scattering (DVCS) in the forward limit, as suggested in $[4,5]$. Besides DVCS, processes with a light hadron or a quarkonium instead of the real photon in the final state of DVCS can be described with GPDs. With the leading power approximation the amplitudes of the processes are given by convolutions of twist-2 GPDs with perturbative coefficient functions. The properties of twist-2 GPDs have been studied extensively (see the reviews in [6-8]). From twist-2 GPDs one can only obtain the sum of the spin part and the orbitalangular momentum part of quarks or gluons. Recently, it has been shown in [9-12] that one can obtain each orbital-angular momentum part from twist-3 GPDs individually. Therefore, it is important to study twist-3 GPDs and how to extract them from experiment.

In general twist-3 GPDs only appear in power-suppressed contributions. In this work we study the power-suppressed contributions of photoproduction of $J / \psi$ or $\Upsilon$. The amplitude at the leading power of $J / \psi$ - or $\Upsilon$ photoproduction has been given in [15]. At the leading power, only a part of gluonic twist-2 GPDs is involved. The produced quarkonium at twist-2 level is only transversely polarized. At the next-toleading power, the quarkonium is longitudinally polarized. If one can measure the polarization, then twist-3 GPDs can be accessed directly. It is interesting to note that the production rate of longitudinally polarized $J / \psi$ or $\Upsilon$ can be large because the polarization vector is proportional to the energy in contrast to the transverse polarization vectors which are constant. This can enhance the power-suppressed contributions in the high energy limit. This motivates us to study the process at twist-3 level in this work. After we have completed the power-suppressed contributions of photoproduction of $J / \Psi$ in terms of twist-3 GPDs, we should take care of an important property of the GPDs to ensure that these calculations are safe. This property has been shown in a series 
of papers [16-23], which tell that some twist-3 GPDs may be discontinuous. We will discuss this at the end of our calculation.

\section{Definitions and properties of GPDs}

We consider the process

$\gamma(q)+h(p) \rightarrow J_{Q}(k)+h\left(p^{\prime}\right)$,

where the quarkonium is denoted as $J_{Q}$. We will consider the case where $J_{Q}$ is a ${ }^{3} S_{1}$ state of a $Q \bar{Q}$ state with $Q=c, b$ or a ${ }^{1} P_{1}$ state. The ${ }^{3} S_{1}$ state corresponds to $J / \psi$ or $\Upsilon$, while the ${ }^{1} P_{1}$ state corresponds to $h_{c, b}$. In Eq. (1) the initial or final hadron $h$ is a proton or a spin-1/2 hadron. The kinematical variables of the process are given by $s=(p+q)^{2}$ and $t=\left(p^{\prime}-p\right)^{2}$. This process with $J / \psi$ at leading twist has been studied extensively in, e.g., [13-15] and the references therein.

We will use the light-cone coordinate system, in which a vector $a^{\mu}$ is expressed as $a^{\mu}=\left(a^{+}, a^{-}, \mathbf{a}_{\perp}\right)=\left(\left(a^{0}+\right.\right.$ $\left.\left.a^{3}\right) / \sqrt{2},\left(a^{0}-a^{3}\right) / \sqrt{2}, a^{1}, a^{2}\right)$ and $a_{\perp}^{2}=\left(a^{1}\right)^{2}+\left(a^{2}\right)^{2}$. We define two light-cone vectors $n^{\mu}=(0,1,0,0)$ and $l^{\mu}=$ $(1,0,0,0)$. The transverse metric is given by $g_{\perp}^{\mu \nu}=g^{\mu \nu}-$ $n^{\mu} l^{\nu}-n^{\nu} l^{\mu}$. We will also need the transverse antisymmetric tensor which is given by $\epsilon_{\perp}^{\mu \nu}=\epsilon^{\alpha \beta \mu \nu} l_{\alpha} n_{\beta}$ with $\epsilon_{\perp}^{12}=1$. We take a frame in which the momenta in Eq. (1) are given by

$$
\begin{aligned}
p^{\mu} & =\left(p^{+}, p^{-},-\frac{1}{2} \boldsymbol{\Delta}_{\perp}\right), \\
p^{\prime \mu} & =\left(p^{\prime+}, p^{-}, \frac{1}{2} \boldsymbol{\Delta}_{\perp}\right), \\
q^{\mu} & =\left(q^{+}, q^{-}, \frac{1}{2} \boldsymbol{\Delta}_{\perp}\right),
\end{aligned}
$$

with $\Delta^{\mu}=\left(p^{\prime}-p\right)^{\mu}$. In the forward region the components of $\Delta^{\mu}$ except $\Delta^{+}$are small, they scale like

$$
\Delta_{\perp}^{\mu} \sim Q(\lambda, \lambda), \quad \Delta^{-} \sim p^{-} \sim p^{-} \sim q^{+} \sim Q \lambda^{2}
$$

with $\lambda \ll 1 . Q$ is a generic large scale of the process. It can be the heavy quark mass or $\sqrt{s}$. In this kinematical region the momentum of $J_{Q}$ is

$$
\begin{aligned}
k_{\perp}^{\mu} & =-\frac{1}{2} \Delta_{\perp}^{\mu}, \\
k^{+} & =-\Delta^{+}+\mathcal{O}\left(Q \lambda^{2}\right), \\
k^{-} & =q^{-}+\mathcal{O}\left(Q \lambda^{2}\right) .
\end{aligned}
$$

The produced quarkonium moves almost in the direction of the initial photon.

For the process QCD factorization is expected in which the nonperturbative effect related to the quarkonium is included in matrix elements of non-relativistic QCD (NRQCD) and that of the initial hadron is included in various GPDs. The produced quarkonium mainly consists of a heavy quark $Q$ and a heavy antiquark $\bar{Q}$. The relative velocity $v$ between $Q$ and $\bar{Q}$ inside the quarkonium is small. One can make a small velocity expansion and use NRQCD factorization in [24] for the quarkonium. In general, a quarkonium is a bound state of a heavy quark $Q \bar{Q}$ pair combined with light partons, i.e., a quarkonium state is a superposition of a $Q \bar{Q}$ state and states of $Q \bar{Q}$ combined with gluons and light-quark pairs. We take the leading order of $v$. At this order quarkonium, considered in this work, can be taken only as a bound state of $Q \bar{Q}$. The other components with light partons, like the $Q \bar{Q} g$ component, contribute only at the higher orders in the small velocity expansion according to the power counting in [24].

For our calculation one can use a wave function $\psi$ to project the relevant state of the produced $Q \bar{Q}$ pair. The projection is standard. For $J / \psi$ or $\Upsilon$ the derived amplitude will be proportional to the wave function at the origin. The amplitude of $h_{c}$ is proportional to the derivative of the corresponding wave function at the origin. In this work we use the notation of wave functions. It should be noted that the wave function at the origin or the derivative are in fact the corresponding NRQCD matrix elements defined in [24]. The relations can be found in [24]. It will be important to express our results with these NRQCD matrix elements to perform NRQCD factorization beyond tree level. The Coulomb singularities appearing at higher orders of $\alpha_{s}$ are factorized into NRQCD matrix elements as shown in [24]. In terms of wave functions, it is also noted that the effects of the detailed shapes of the wave functions are included in those NRQCD matrix elements at higher orders of the small velocity expansion. In this work we take the leading order of $v$ and $\alpha_{s}$.

Before analyzing the process, we introduce twist-3 parton GPDs and discuss their properties. We define the momentum $\bar{P}$ and the variable $\xi$ by

$\bar{P}=\frac{p+p^{\prime}}{2}, \quad \xi=\frac{p^{+}-p^{\prime+}}{p^{+}+p^{\prime+}}=\frac{-\Delta \times n}{2 \bar{P} \times n}$.

The twist-3 gluonic GPDs are given by the following matrix elements:

$$
\begin{aligned}
& M_{D}^{\mu \nu \rho}\left(x_{1}, x_{2}, \Delta\right) \\
& =\int \frac{\mathrm{d} \lambda_{1} \mathrm{~d} \lambda_{2}}{(2 \pi)^{2}} \mathrm{e}^{i \bar{P}^{+} \lambda_{1}\left(x_{1}+x_{2}\right) / 2+i \bar{P}^{+} \lambda_{2}\left(x_{2}-x_{1}\right)} \\
& \left\langle p^{\prime}, \lambda^{\prime}\left|G^{a,+\mu}\left(-\frac{\lambda_{1} n}{2}\right) \tilde{D}_{\perp a b}^{\nu}\left(\lambda_{2} n\right) \hat{G}^{b,+\rho}\left(\frac{\lambda_{1} n}{2}\right)\right| p, \lambda\right\rangle, \\
& M_{F}^{\mu \nu \rho}\left(x_{1}, x_{2}, \Delta\right) \\
& =\text { if }^{a b c} \frac{g_{s}}{\bar{P}^{+}} \int \frac{\mathrm{d} \lambda_{1} \mathrm{~d} \lambda_{2}}{(2 \pi)^{2}} \mathrm{e}^{i \bar{P}^{+} \lambda_{1}\left(x_{1}+x_{2}\right) / 2+i \bar{P}^{+} \lambda_{2}\left(x_{2}-x_{1}\right)} \\
& \quad \times\left\langle p^{\prime}\left|G^{a,+\mu}\left(-\frac{\lambda_{1} n}{2}\right) G^{b,+v}\left(\lambda_{2} n\right) G^{c,+\rho}\left(\frac{\lambda_{1} n}{2}\right)\right| p\right\rangle,
\end{aligned}
$$




$$
\begin{aligned}
& M_{\partial}^{\mu \nu \rho}(x, \Delta)=\frac{1}{\bar{P}^{+}} \int \frac{\mathrm{d} \lambda}{2 \pi} \mathrm{e}^{i \bar{P}^{+} \lambda x} \\
& \quad \times\left\langle p^{\prime}, \lambda^{\prime}\left|G^{a,+\mu}\left(-\frac{\lambda n}{2}\right) \tilde{\partial}^{v} G^{a,+\rho}\left(\frac{\lambda n}{2}\right)\right| p, \lambda\right\rangle,
\end{aligned}
$$

where the derivative $\tilde{\partial}^{\mu}$ is defined by

$$
f^{\dagger}(x) \tilde{\partial}^{\mu} g(x)=\frac{1}{2}\left[f^{\dagger}(x) \partial^{\mu} g(x)-\left(\partial^{\mu} f(x)\right)^{\dagger} g(x)\right]
$$

for arbitrary functions $f(x)$ and $g(x)$. The covariant derivative $D^{\mu}$ is given by $D^{\mu}(x)=\partial^{\mu}+i g_{s} G^{\mu}(x) . \tilde{D}^{\mu}$ is defined with $D^{\mu}$ in a similar way to $\tilde{\partial}^{\mu}$. All Lorentz indices $\mu, v$ and $\rho$ are transverse. There is another twist- 3 matrix element of gluonic GPDs, which is obtained by replacing $f^{a b c}$ in $M_{F}^{\mu \nu \rho}\left(x_{1}, x_{2}, \Delta\right)$ by $d^{a b c}$. This matrix element is irrelevant for the process in Eq. (1). But it will be relevant for the process when the produced quarkonium has the quantum number $C=+$.

The definitions in Eq. (6) are given in the light-cone gauge $n \times G=0$. In other gauges one has to insert gauge links between field operators to make the definitions gauge invariant. These gauge links are built with

$$
\mathcal{L}(x)=P \exp \left\{-i g_{s} \int_{0}^{\infty} \mathrm{d} \lambda G^{+}(\lambda n+x)\right\} .
$$

It is noted that in gauges other than the light-cone one, the definition of $M_{\partial}^{\mu \nu \rho}$ is given by replacing $\tilde{\partial}^{v}$ with $\mathcal{L}^{\dagger}(-\lambda n / 2) \tilde{\partial}^{v} \mathcal{L}(\lambda n / 2)$ in Eq. (6). Because the derivative also acts on gauge links, the symmetry of time reversal does not give any constraint for $M_{\partial}^{\mu \nu \rho}$. The defined matrix elements are not independent. Using the identity

$$
\begin{aligned}
& \mathcal{L}(y n) D_{\perp}^{\mu}(y n) \mathcal{L}^{\dagger}(y n) F \\
& \quad=\partial_{\perp}^{\mu} F-i g_{s} \int_{0}^{\infty} \mathrm{d} \lambda\left(\mathcal{L} G^{+\mu} \mathcal{L}^{\dagger}\right)((\lambda+y) n) F,
\end{aligned}
$$

for an arbitrary function $F$ one can derive the relation

$$
\begin{aligned}
& M_{D}^{\mu v \rho}\left(x_{1}, x_{2}, \Delta\right) \\
& \quad=\frac{M_{F}^{\mu \nu \rho}\left(x_{1}, x_{2}, \Delta\right)}{x_{1}-x_{2}+i \varepsilon}+\delta\left(x_{2}-x_{1}\right) M_{\partial}^{\mu \nu \rho}\left(x_{1}, \Delta\right),
\end{aligned}
$$

where the factor $i \varepsilon$ in the denominator appears because the integration over $\lambda$ in the identity in Eq. (9) is from 0 to $\infty$. Therefore, only two of the three matrix elements in Eq. (6) are independent.

In general, each matrix element with three transverse indices can be parameterized with scalar functions or GPDs. By taking parity symmetry into account the number of such functions of each matrix element cannot be larger than 16 for a spin-1/2 hadron. However, in this work, we will only encounter those matrix elements with two indices contracted with $g_{\perp}^{\mu \nu}$. There are three possible contractions. We consider first the contraction with the first and third Lorentz index. We define the contracted matrix elements and give their parameterization by

$$
\begin{aligned}
G_{F, D}^{\mu}\left(x_{1}, x_{2}, \Delta\right)= & g_{\perp v \rho} M_{F, D}^{\rho \mu \nu}\left(x_{1}, x_{2}, \Delta\right), \\
G_{\partial}^{\mu}(x, \Delta)= & g_{\perp v \rho} M_{\partial}^{\rho \mu \nu}(x, \Delta), \\
G_{\partial}^{\mu}(x, \Delta)= & \bar{u}\left(p^{\prime}\right)\left[i \gamma^{+} \frac{\Delta_{\perp}^{\mu}}{\bar{P}^{+}} H_{1}+\frac{\sigma^{+\mu} m}{\bar{P}+} H_{2}\right. \\
& +\frac{\Delta_{\perp \rho}}{\bar{P}^{+}} \epsilon^{+-\mu \rho} \gamma^{+} \gamma_{5} H_{3} \\
& \left.+\frac{\Delta_{\perp}^{\mu} \sigma^{+\rho} \Delta_{\perp \rho}}{m \bar{P}^{+}} H_{4}\right] u(p), \\
G_{F}^{\mu}\left(x_{1}, x_{2}, \Delta\right)= & \bar{u}\left(p^{\prime}\right)\left[i \gamma^{+} \frac{\Delta_{\perp}^{\mu}}{\bar{P}^{+}} G_{1}+\frac{\sigma^{+\mu} m}{\bar{P}^{+}} G_{2}\right. \\
& +\frac{\Delta_{\perp \rho}}{\bar{P}^{+}} \epsilon^{+-\mu \rho} \gamma^{+} \gamma_{5} G_{3} \\
& \left.+\frac{\Delta_{\perp}^{\mu} \sigma^{+\rho} \Delta_{\perp \rho}}{m \bar{P}^{+}} G_{4}\right] u(p), \\
G_{D}^{\mu}\left(x_{1}, x_{2}, \Delta\right)= & \bar{u}\left(p^{\prime}\right)\left[i \gamma^{+} \frac{\Delta_{\perp}^{\mu}}{\bar{P}^{+}} F_{1}+\frac{\sigma^{+\mu} m}{\bar{P}^{+}} F_{2}\right. \\
& +\frac{\Delta_{\perp \rho}}{\bar{P}^{+} \epsilon^{+-\mu \rho} \gamma^{+} \gamma_{5} F_{3}} \\
& \left.+\frac{\Delta_{\perp}^{\mu} \sigma^{+\rho} \Delta_{\perp \rho}}{m \bar{P}^{+}} F_{4}\right] u(p),
\end{aligned}
$$

where the scalar functions $G_{i}, F_{i}$ and $H_{i}$ depend on momentum fractions, $\xi$ and $t$. There is a freedom by choosing different parameterizations. The parametrization in the above has the advantage that all spinor products $\bar{u}\left(p^{\prime}\right) \Gamma u(p)$ with $\Gamma=\gamma^{+}, \sigma^{+\mu}$ and $\gamma^{+} \gamma_{5}$ are at the order of $\mathcal{O}\left(\lambda^{0}\right)$. There are constraints for these functions or GPDs from hermiticity, Bose symmetry and time-reversal symmetry. All $G_{i}$ and $F_{i}$ are real and have the properties

$$
\begin{aligned}
& G_{3}\left(x_{1}, x_{2}, \xi, t\right)=-G_{3}\left(x_{2}, x_{1},-\xi, t\right), \\
& G_{1,2,4}\left(x_{1}, x_{2}, \xi, t\right)=G_{1,2,4}\left(x_{2}, x_{1},-\xi, t\right), \\
& F_{3}\left(x_{1}, x_{2}, \xi, t\right)=F_{3}\left(x_{2}, x_{1},-\xi, t\right), \\
& F_{1,2,4}\left(x_{1}, x_{2}, \xi, t\right)=-F_{1,2,4}\left(x_{2}, x_{1},-\xi, t\right), \\
& G_{i}\left(x_{1}, x_{2}, \xi, t\right)=-G_{i}\left(-x_{2},-x_{1}, \xi, t\right), \\
& F_{i}\left(x_{1}, x_{2}, \xi, t\right)=-F_{i}\left(-x_{2},-x_{1}, \xi, t\right), i=1,2,3,4 .
\end{aligned}
$$

It is noted that time-reversal symmetry does not give any constraint for $G_{\partial}^{\mu}$. The functions $H_{i}$ are complex in general. The properties for $H_{i}$ are

$$
\begin{aligned}
H_{1,2,4}^{*}(x, \xi, t) & =-H_{1,2,4}(x,-\xi, t), \\
H_{3}^{*}(x, \xi, t) & =H_{3}(x,-\xi, t), \\
H_{1,2,3,4}(x, \xi, t) & =-H_{1,2,3,4}(-x, \xi, t) .
\end{aligned}
$$

By using the identity

$\frac{1}{x_{1}-x_{2}+i \varepsilon}=P \frac{1}{x_{1}-x_{2}}-i \pi \delta\left(x_{1}-x_{2}\right)$ 
with $P$ standing for the principal-value prescription and by noting that $F_{1,2,3,4}$ and $G_{1,2,3,4}$ are real and $H_{1,2,3,4}$ are complex, we obtain the relations among these GPDs from the relation in Eq. (10):

$$
\begin{aligned}
F_{i}\left(x_{1}, x_{2}, \xi, t\right)= & P \frac{1}{x_{1}-x_{2}} G_{i}\left(x_{1}, x_{2}, \xi, t\right) \\
& +\delta\left(x_{2}-x_{1}\right) \operatorname{Re} H_{i}\left(x_{1}, \xi, t\right), \\
G_{i}(x, x, \xi, t)= & \frac{1}{\pi} \operatorname{Im} H_{i}(x, \xi, t) .
\end{aligned}
$$

In the limit of $\Delta^{\mu} \rightarrow 0$, the GPD $G_{2}, F_{2}$ and $H_{2}$ become gluonic ETQS matrix elements relevant for single transversespin asymmetries in inclusive processes [25-29]. An interesting observation of the limit is made in [9-12]: one of the moments of $F_{3}$ is the orbital-angular momentum contribution of gluons and that of $\mathrm{H}_{3}$ can be interpreted as the canonical orbital-angular momentum contribution in the light-cone gauge as discussed in [30]. It is noticed from Eqs. $(12,15)$ that $H_{3}$ becomes real in the limit by assuming that $G_{3}$ is a continuous function of $\xi$.

The matrix elements contracted in Eq. (6) with $g_{\perp \mu \nu}$ or $g_{\perp v \rho}$ are not independent. We have from Bose symmetry

$$
\begin{aligned}
g_{\perp v \rho} M_{D}^{\mu \nu \rho}\left(x_{1}, x_{2}, \Delta\right) & =-g_{\perp v \rho} M_{D}^{v \rho \mu}\left(-x_{2},-x_{1}, \Delta\right), \\
g_{\perp v \rho} M_{\partial}^{\mu \nu \rho}(x, \Delta) & =-g_{\perp v \rho} M_{\partial}^{v \rho \mu}(-x, \Delta) .
\end{aligned}
$$

Since $M_{F}^{\mu \nu \rho}$ is defined with three field-strength-tensor operators, all three contractions give essentially the same GPDs,

$$
\begin{aligned}
& g_{\perp v \rho} M_{F}^{\mu \nu \rho}\left(x_{1}, x_{2}, \Delta\right) \\
= & -g_{\perp v \rho} M_{F}^{v \rho \mu}\left(-x_{2},-x_{1}, \Delta\right) \\
= & -g_{\perp v \rho} M_{F}^{v \mu \rho}\left(x_{1}, x_{1}-x_{2}+\xi, \Delta\right) .
\end{aligned}
$$

We denote the matrix elements and parameterize them as

$$
\begin{aligned}
\tilde{G}_{D}^{\mu}\left(x_{1}, x_{2}, \Delta\right)= & g_{\perp \rho \nu} M_{D}^{\mu \rho v}\left(x_{1}, x_{2}, \Delta\right) \\
\tilde{G}_{\partial}^{\mu}(x, \Delta)= & g_{\rho v} M_{\partial}^{v \rho \mu}(x, \Delta), \\
\tilde{G}_{\partial}^{\mu}(x, \Delta)= & \bar{u}\left(p^{\prime}\right)\left[i \gamma^{+} \frac{\Delta_{\perp}^{\mu}}{\bar{P}^{+}} \tilde{H}_{1}+\frac{\sigma^{+\mu} m}{\bar{P}^{+}} \tilde{H}_{2}\right. \\
& +\frac{\Delta_{\perp \rho}}{\bar{P}^{+}} \epsilon^{+-\mu \rho} \gamma^{+} \gamma_{5} \tilde{H}_{3} \\
& \left.+\frac{\Delta_{\perp}^{\mu} \sigma^{+\rho} \Delta_{\perp \rho}}{m \bar{P}^{+}} \tilde{H}_{4}\right] u(p), \\
\tilde{G}_{D}^{\mu}\left(x_{1}, x_{2}, \Delta\right)= & \bar{u}\left(p^{\prime}\right)\left[i \gamma^{+} \frac{\Delta_{\perp}^{\mu}}{\bar{P}^{+}} \tilde{F}_{1}+\frac{\sigma^{+\mu} m}{\bar{P}^{+}} \tilde{F}_{2}\right. \\
& +\frac{\Delta_{\perp \rho}}{\bar{P}^{+}} \epsilon^{+-\mu \rho} \gamma^{+} \gamma_{5} \tilde{F}_{3} \\
& \left.+\frac{\Delta_{\perp}^{\mu} \sigma^{+\rho} \Delta_{\perp \rho}}{m \bar{P}^{+}} \tilde{F}_{4}\right] u(p) .
\end{aligned}
$$

From the symmetries, the functions $\tilde{F}_{i}$ are real and the functions $\tilde{H}_{i}$ are complex. They have the properties

$$
\begin{aligned}
\tilde{F}_{3}\left(x_{1}, x_{2}, \xi, t\right) & =-\tilde{F}_{3}\left(-x_{1},-x_{2},-\xi, t\right), \\
\tilde{F}_{1,2,4}\left(x_{1}, x_{2}, \xi, t\right) & =\tilde{F}_{1,2,4}\left(-x_{1},-x_{2},-\xi, t\right), \\
\tilde{H}_{3}^{*}(x, \xi, t) & =-\tilde{H}_{3}(-x,-\xi, t), \\
\tilde{H}_{1,2,4}^{*}(x, \xi, t) & =\tilde{H}_{1,2,4}(-x,-\xi, t) .
\end{aligned}
$$

Again, these functions are not independent. They satisfy the relations

$$
\begin{aligned}
& \tilde{F}_{i}\left(x_{1}, x_{2}, \xi, t\right)=-P \frac{1}{x_{1}-x_{2}} G_{i}\left(x_{1}, x_{1}-x_{2}+\xi, \xi, t\right) \\
& \quad+\delta\left(x_{2}-x_{1}\right) \operatorname{Re} \tilde{H}_{i}(x, \xi, t), \\
& G_{i}\left(x_{1}, x_{1}-x_{2}+\xi, \xi, t\right)=-\frac{1}{\pi} \operatorname{Im} \tilde{H}_{i}(x, \xi, t),
\end{aligned}
$$

for $i=1,2,3,4$. These relations can be derived from Eq. (10) similarly to those in Eq. (15).

Twist-3 matrix elements with quarks also appear in the twist-3 amplitudes of the considered processes at tree level. There are three matrix elements:

$$
\begin{aligned}
& N_{F}^{v}\left(x_{1}, x_{2}, \Delta\right) \\
& =\int \frac{\mathrm{d} \lambda_{1}}{2 \pi} \frac{\mathrm{d} \lambda_{2}}{2 \pi} e^{i \bar{P}^{+} \lambda_{1}\left(x_{1}+x_{2}\right) / 2+i \bar{P}^{+} \lambda_{2}\left(x_{2}-x_{1}\right)} \\
& \quad \times\left\langle p^{\prime}\left|\bar{\psi}\left(-\lambda_{1} n / 2\right) \gamma^{+} g_{s} G^{+v}\left(\lambda_{2} n\right) \psi\left(\lambda_{1} n / 2\right)\right| p\right\rangle, \\
& N_{D}^{v}\left(x_{1}, x_{2}, \Delta\right) \\
& =\bar{P}^{+} \int \frac{\mathrm{d} \lambda_{1}}{2 \pi} \frac{\mathrm{d} \lambda_{2}}{2 \pi} \mathrm{e}^{i \bar{P}^{+} \lambda_{1}\left(x_{1}+x_{2}\right) / 2+i \bar{P}^{+} \lambda_{2}\left(x_{2}-x_{1}\right)} \\
& \quad \times\left\langle p^{\prime}\left|\bar{\psi}\left(-\lambda_{1} n / 2\right) \gamma^{+} \tilde{D}_{\perp}^{v}\left(\lambda_{2} n\right) \psi\left(\lambda_{1} n / 2\right)\right| p\right\rangle, \\
& N_{\partial}^{v}(x, \Delta) \\
& =\int \frac{d \lambda}{2 \pi} \mathrm{e}^{i \bar{P}^{+} \lambda x}\left\langle p^{\prime}\left|\bar{\psi}(-\lambda n / 2) \gamma^{+} \tilde{\partial}_{\perp}^{v} \psi(\lambda n / 2)\right| p\right\rangle .
\end{aligned}
$$

Similarly, one can derive the relation between these three matrix elements by using the identity in Eq. (9):

$$
\begin{aligned}
N_{D}^{v}\left(x_{1}, x_{2}, \Delta\right)= & \frac{1}{x_{1}-x_{2}+i \varepsilon} N_{F}^{v}\left(x_{1}, x_{2}, \Delta\right) \\
& +\delta\left(x_{2}-x_{1}\right) N_{\partial}^{v}\left(x_{1}, \Delta\right) .
\end{aligned}
$$

A similar relation has also been derived in $[11,12]$, where $i \varepsilon$ in the denominator is missing. In our work, only the sum of all quark flavors is relevant. We define the following quarkgluon GPDs: 


$$
\begin{aligned}
\sum_{q} N_{F}^{\mu}\left(x_{1}, x_{2}\right)= & \bar{u}\left(p^{\prime}\right)\left[i \gamma^{+} \frac{\Delta_{\perp}^{\mu}}{\bar{P}^{+}} N_{1}+\frac{\sigma^{+\mu} m}{\bar{P}^{+}} N_{2}\right. \\
& +\frac{\Delta_{\perp \rho}}{\bar{P}^{+}} \epsilon^{+-\mu \rho} \gamma^{+} \gamma_{5} N_{3} \\
& \left.+\frac{\Delta_{\perp}^{\mu} \sigma^{+\rho} \Delta_{\perp \rho}}{m \bar{P}^{+}} N_{4}\right] u(p) .
\end{aligned}
$$

The sum is over the light flavors of quarks. In the limit of $\Delta^{\mu} \rightarrow 0$, one of the moments of $N_{3}$ is the orbital-angular momentum contribution of quarks as observed in [9-12].

For the completeness of the gluon GPDs, we also include the definitions of gluonic twist-2 GPDs. These GPDs have been discussed in detail in [7,8]. They will appear in our amplitudes. We take the notation of [7] for twist-2 gluon GPDs:

$$
\begin{aligned}
& \mathcal{F}_{g}=\frac{1}{\bar{P}^{+}} \int \frac{\mathrm{d} \lambda}{2 \pi} e^{i x \bar{P}^{+} \lambda} \\
& \times\left\langle p^{\prime}\left|g_{\perp \mu \nu} G^{+\mu}\left(-\frac{1}{2} \lambda n\right) G^{+v}\left(\frac{1}{2} \lambda n\right)\right| p\right\rangle \\
& =-\frac{1}{2 \bar{P}^{+}} \bar{u}\left(p^{\prime}\right)\left[\gamma^{+} H_{g}(x, \xi, t)\right. \\
& \left.+\frac{i \sigma^{+\alpha}}{2 m} \Delta_{\alpha} E_{g}(x, \xi, t)\right] u(p), \\
& \tilde{\mathcal{F}}_{g}=\frac{i}{\bar{P}^{+}} \int \frac{\mathrm{d} \lambda}{2 \pi} \mathrm{e}^{i x \bar{P}^{+} \lambda} \\
& \times\left\langle p^{\prime}\left|\epsilon_{\perp \mu \nu} G^{+\mu}\left(-\frac{1}{2} \lambda n\right) G^{+v}\left(\frac{1}{2} \lambda n\right)\right| p\right\rangle \\
& =-\frac{1}{2 \bar{P}^{+}} \bar{u}\left(p^{\prime}\right)\left[\gamma^{+} \gamma_{5} \tilde{H}_{g}(x, \xi, t)\right. \\
& \left.+\frac{\gamma_{5} \Delta^{+}}{2 m} \tilde{E}_{g}(x, \xi, t)\right] u(p) \\
& \mathcal{F}_{T g}^{\mu \nu}=\frac{1}{2 \bar{P}^{+}} \int \frac{\mathrm{d} \lambda}{2 \pi} e^{i x \bar{P}^{+} \lambda} \\
& \times\left\langle p^{\prime}\left|\mathbf{S}\left(G^{+\mu}\left(-\frac{1}{2} \lambda n\right) G^{+v}\left(\frac{1}{2} \lambda n\right)\right)\right| p\right\rangle \\
& =-\frac{1}{2 \bar{P}^{+}} \mathbf{S}\left\{\frac{\bar{P}^{+} \Delta^{v}-\Delta^{+} \bar{P}^{v}}{2 m \bar{P}^{+}}\right. \\
& \times \bar{u}\left(p^{\prime}\right)\left[i \sigma^{+\mu} H_{g T}(x, \xi, t)\right. \\
& +\frac{\bar{P}^{+} \Delta^{\mu}-\Delta^{+} \bar{P}^{\mu}}{m^{2}} \tilde{H}_{g T}(x, \xi, t) \\
& +\frac{\gamma^{+} \Delta^{\mu}-\Delta^{+} \gamma^{\mu}}{2 m} E_{g T}(x, \xi, t) \\
& \left.\left.+\frac{\gamma^{+} \bar{P}^{\mu}-\bar{P}^{+} \gamma^{\mu}}{m} \tilde{E}_{g T}(x, \xi, t)\right] u(p)\right\},
\end{aligned}
$$

where $\mu$ and $v$ are transverse. The notation $\mathbf{S}(\cdots)$ implies that the tensors in $(\cdots)$ are symmetric and traceless. There

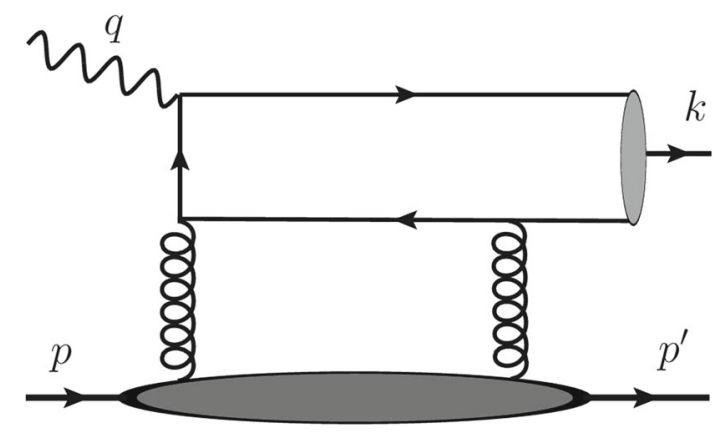

(a)

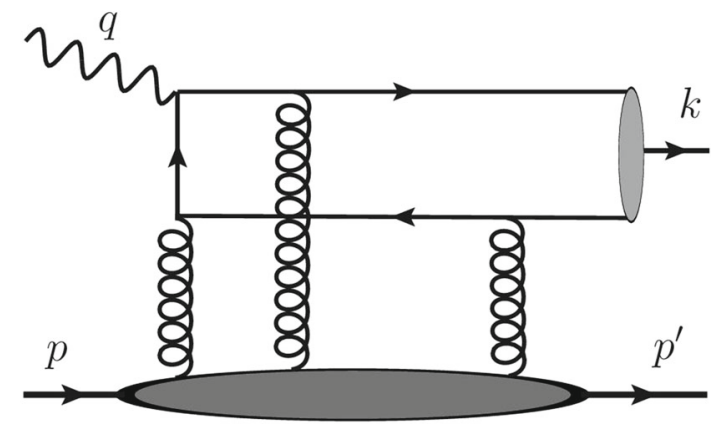

(b)

Fig. 1 a One of the six diagrams for two-gluon exchanges. b One diagrams for three-gluon exchanges. Other diagrams are obtained through permutation

are in total eight twist-2 gluon GPDs. Their properties can be found in $[7,8]$.

\section{Calculations for $J / \Psi$ Production}

Now we turn to our derivation of the twist-2- and twist-3 amplitudes for the process in Eq. (1) at tree level in the case that the quarkonium is a ${ }^{3} S_{1} Q \bar{Q}$ state. We will perform the calculation mainly in the light-cone gauge $n \times G=G^{+}=0$, but also partly in Feynman gauge for fixing some ambiguities. At tree level, the quarkonium is produced through exchanges of gluons between the heavy quark pair and the initial hadron. The two-gluon- or three-gluon exchanges are illustrated in Fig. 1a or b, respectively. After projecting out the ${ }^{3} S_{1}$ state from the produced $Q \bar{Q}$ pair, the contribution from Fig. 1a can be written as

$$
\begin{aligned}
\left.i \mathcal{T}\right|_{1 a} & =\frac{1}{2} \int \mathrm{d}^{4} k_{1} \mathrm{~d}^{4} k_{2} \mathcal{M}^{\mu \nu}\left(k_{1}, k_{2}, \Delta\right) \\
& \times \int \frac{\mathrm{d}^{4} \xi_{1}}{(2 \pi)^{4}} \frac{\mathrm{d}^{4} \xi_{2}}{(2 \pi)^{4}} e^{i \xi_{1} \times k_{1}+i \xi_{2} k_{2}}\left\langle p^{\prime}\left|G_{\mu}\left(\xi_{1}\right) G_{v}\left(\xi_{2}\right)\right| p\right\rangle
\end{aligned}
$$

where $\mathcal{M}^{\mu v}\left(k_{1}, k_{2}, \Delta\right)$ is essentially the amplitude of $\gamma(q)+$ $g\left(k_{1}\right)+g\left(k_{2}\right) \rightarrow J_{Q}(k)$ after taking the trace of colors. It is given by six diagrams. To make a power-expansion related to 
the light hadron, one notes that in the forward limit the gauge fields in the matrix element of Eq. (26) and the momenta carried by these fields scale like

$$
G^{\mu} \sim Q\left(1, \lambda^{2}, \lambda, \lambda\right), \quad k_{1,2}^{\mu} \sim Q\left(1, \lambda^{2}, \lambda, \lambda\right) .
$$

In the limit, the momentum transfer $\Delta^{\mu}$ also scales like $\Delta^{\mu} \sim Q\left(1, \lambda^{2}, \lambda, \lambda\right)$. The twist-2 or leading power contribution of the amplitude is obtained by taking the leading order of the expansion in $\lambda$. For this purpose we need to expand $\mathcal{M}^{\mu \nu}\left(k_{1}, k_{2}, \Delta\right)$ and the matrix element in $\lambda$. In the lightcone gauge, the leading contribution of the matrix element is given when the two-gluon fields carry transverse indices, i.e., $\mu, v=\perp . \mathcal{M}^{\mu \nu}\left(k_{1}, k_{2}, \Delta\right)$ at the leading order for $\mu, v=\perp$ is given by

$$
\begin{aligned}
\mathcal{M}^{\mu \nu}\left(k_{1}, k_{2}, \Delta\right)= & i g_{s}^{2} A_{J}\left[\frac{16 m_{Q}^{2}}{k^{+} q^{-}} \frac{k_{1}^{+} k_{2}^{+}}{\left(k_{2}^{+}-i \varepsilon\right)\left(k_{1}^{+}-i \varepsilon\right)}\right. \\
& \left.\times g_{\perp}^{\mu \nu} \epsilon_{\perp}(q) \times \epsilon^{*}(k)\right]\left(1+\mathcal{O}\left(\lambda^{2}\right)\right) \\
A_{J}= & Q_{Q} e \psi^{*}(0) \frac{\left(2 m_{Q}\right)^{-3 / 2}}{\sqrt{N_{c}}},
\end{aligned}
$$

where $\psi(0)$ is the non-relativistic wave function at the origin. $\epsilon(q)$ and $\epsilon(k)$ are the polarization vectors of the photon and $J_{Q}$, respectively. The factor $i \varepsilon$ in the denominator comes from the quark propagators in Fig. 1a. In the gauge $n \times G=0$, the twist- 2 contribution comes only from twogluon exchanges as in Fig. 1a. It is straightforward to obtain the twist-2 amplitude denoted as $\mathcal{T}_{T}$ :

$$
\begin{aligned}
\mathcal{T}_{T}= & 8 g_{s}^{2} A_{J} \epsilon_{\perp}(q) \times \epsilon^{*}(k) \\
& \times \int \mathrm{d} x \frac{1}{(x-\xi+i \varepsilon)(x+\xi-i \varepsilon)} \mathcal{F}_{g}(x, \xi, t) .
\end{aligned}
$$

This result is in agreement with that given in [15]. We note here that at twist- 2 the produced $J / \psi$ or $\Upsilon$ is transversely polarized. Using this amplitude one can extract from experimental data only the GPD $H_{g}$ and $E_{g}$ contained in $\mathcal{F}_{g}$.

Now we consider the amplitude with longitudinally polarized $J / \psi$ or $\Upsilon$. This amplitude is power-suppressed. In contrast to the twist- 2 amplitude $\mathcal{T}_{T}$, there are several sources of contributions. There are contributions which involve twist-2 gluon GPDs and are explicitly power-suppressed. They are called dynamical twist-3 contributions. There are also contributions which involve twist-3 GPDs. One part of dynamical twist- 3 contributions can easily be identified from Eq. (29). In the used frame given by Eq. (2), the polarization vector $\epsilon_{L}$ of the longitudinally polarized $J / \psi$ has a small transverse part at the order of $\lambda$. To specify the polarization of $J / \psi$, one has to define the spin quantization axis properly. We take the socalled $s$-channel helicity frame by introducing the 4 -vector $X^{\mu}=q+p$. The polarization vector $\epsilon_{L}^{\mu}$ of this frame is given by

$\epsilon_{L}^{\mu}(k)=\frac{k \times X}{\sqrt{(k \times X)^{2}-M^{2} s}}\left(\frac{k^{\mu}}{M}-\frac{M}{k \cdot X} X^{\mu}\right)$.

The vector $\epsilon_{L}$ given here is covariant with $k^{2}=M^{2}$. The physical meaning of the vector $X^{\mu}$ is that in the rest frame of $J_{Q}$ the spin quantization direction is the direction of the 3 -vector $-\mathbf{X}$. Keeping the leading order of $\lambda$, we have

$$
\begin{aligned}
\epsilon_{L}^{\mu} & =\left(\epsilon_{L}^{+}, \epsilon_{L}^{-}, 0,0\right)+\epsilon_{\perp}^{\mu}+\mathcal{O}\left(\lambda^{2}\right), & \epsilon_{L}^{-} & =\frac{k^{-}}{2 m_{Q}}, \\
\epsilon_{L \perp}^{\mu} & =\frac{\rho_{L}}{2 m_{Q}} k_{\perp}^{\mu}, & \rho_{L} & =\frac{s+4 m_{Q}^{2}}{s-4 m_{Q}^{2}} .
\end{aligned}
$$

The component $\epsilon_{L}^{+}$is not needed to express our result. Given the $\epsilon_{L \perp}^{\mu}$, we obtain the part of dynamical twist-3 contribution from the transverse part of $\epsilon_{L}^{\mu}$ which can be read off from Eq. (29):

$$
\begin{aligned}
\left.\mathcal{T}\right|_{a, 30}= & -g_{s}^{2} A_{J} \frac{2}{m_{Q}} \epsilon_{\perp}(q) \cdot \Delta_{\perp} \rho_{L} \\
& \times \int d x \frac{1}{(x-\xi+i \varepsilon)(x+\xi-i \varepsilon)} \mathcal{F}_{g}(x, \xi, t),
\end{aligned}
$$

which is power-suppressed, as indicated by the factor $\Delta_{\perp}$.

In the gauge $n \cdot G=0$ the remaining twist- 3 contributions come from two-gluon and three-gluon exchanges as the diagrams in Fig.1. We first study the contributions from Fig. 1a. It is nontrivial to find the twist-3 contributions from Fig. 1a because Fig. 1a also has the leading or twist-2 contributions. With the power counting given in Eq. (27), one part of the twist-3 contributions is given when one of the twogluon fields carries the --index. In this case one should take the leading order of the $\lambda$-expansion for $\mathcal{M}^{\mu+}$. Another part comes from the next-to-leading order of the $\lambda$-expansion for $\mathcal{M}^{\mu v}$ with $\mu=v=\perp$. We denote this contribution of $\mathcal{M}^{\mu+}$ by $\mathcal{M}_{1}^{\mu \nu}$. After the expansion of $\mathcal{M}^{\mu \nu}$ we have

$$
\begin{aligned}
\mathcal{M}^{\mu+}\left(k_{1}, k_{2}, \Delta\right)= & \mathcal{M}^{+\mu}\left(k_{1}, k_{2}, \Delta\right) \\
= & i g_{s}^{2} A_{J} \epsilon^{\mu}\left[\frac{-16 m_{Q}^{2} \epsilon_{L}^{-} k_{1}^{+} k_{2}^{+}}{q^{-} k^{-}\left(k_{2}^{+}-i \varepsilon\right)\left(k_{1}^{+}-i \varepsilon\right)}\right] \\
& (1+\mathcal{O}(\lambda)), \\
\mathcal{M}_{1}^{\mu \nu}\left(k_{1}, k_{2}, \Delta\right)= & i g_{s}^{2} A_{J}\left[\frac{16 m_{Q}^{2} \epsilon_{L}^{-} \epsilon_{\rho}}{k^{+} q^{-} k^{-}\left(k_{2}^{+}-i \varepsilon\right)\left(k_{1}^{+}-i \varepsilon\right)}\right. \\
& \left(g_{\perp}^{\mu \nu} k_{1 \perp}^{\rho}\left(k_{2}^{+}\right)^{2}+g_{\perp}^{\mu \nu} k_{2 \perp}^{\rho}\left(k_{1}^{+}\right)^{2}\right. \\
& -k_{1 \perp}^{\nu} g_{\perp}^{\mu \rho} k_{2}^{+}\left(k_{1}^{+}+k_{2}^{+}\right) \\
& \left.\left.-k_{2 \perp}^{\mu} g_{\perp}^{\nu \rho} k_{1}^{+}\left(k_{1}^{+}+k_{2}^{+}\right)\right)\right],
\end{aligned}
$$


with $\mu, v=\perp$. From $\mathcal{M}^{\mu+}$ we have the twist-3 contribution:

$$
\begin{aligned}
\left.i \mathcal{T}\right|_{a, 31}= & i g_{s}^{2} A_{J} \int \mathrm{d} k_{1}^{+} \mathrm{d} k_{2}^{+}\left[\frac{16 m_{Q}^{2} \epsilon_{L}^{-} \epsilon_{\mu}}{q^{-} k^{-}\left(k_{2}^{+}-i \varepsilon\right)\left(k_{1}^{+}-i \varepsilon\right)}\right] \\
& \times \int \frac{\mathrm{d} \xi_{1}^{-} \mathrm{d} \xi_{2}^{-}}{(2 \pi)^{2}} e^{i \xi_{1}^{-} k_{1}^{+}+i \xi_{2}^{-} k_{2}^{+}} \\
& \times\left\langle p^{\prime}\left|G^{+\mu}\left(\xi_{1}^{-} n\right) G^{+-}\left(\xi_{2}^{-} n\right)\right| p\right\rangle
\end{aligned}
$$

It is noted that the field $G^{-}$is not dynamically independent. From the QCD equation of motion in the gauge $G^{+}=0$ we have the equation for $G^{+-}$:

$$
\begin{aligned}
\partial^{+} G^{a,-+}(x) & =-\left(D_{\perp \mu}(x) G^{\mu+}(x)\right)^{a}+g_{s} J^{a,+}(x), \\
J^{a, \mu}(x) & =\sum_{q} \bar{q}(x) T^{a} \gamma^{\mu} q(x),
\end{aligned}
$$

and the solution is

$$
\begin{aligned}
G^{a,+-}(x)= & i \int \frac{\mathrm{d} \lambda \mathrm{d} k^{+}}{2 \pi} \frac{e^{-i \lambda k^{+}}}{k^{+}+i \varepsilon}\left[\left(g_{s} J^{a,+}(x+\lambda n)\right.\right. \\
& \left.\left.+D_{\perp \mu} G^{+\mu}\right)^{a}(x+\lambda n)\right] .
\end{aligned}
$$

Using this result with a little algebra we derive

$$
\begin{aligned}
\left.i \mathcal{T}\right|_{a, 31}= & g_{s}^{2} A_{J} \frac{16 m_{Q}^{2} \epsilon_{L}^{-} \epsilon_{\rho}}{\left(k^{-}\right)^{2}} \int \mathrm{d} k_{1}^{+} \mathrm{d} k_{2}^{+} \frac{1}{\left(k_{2}^{+}-i \varepsilon\right)^{2}\left(k_{1}^{+}-i \varepsilon\right)} \\
& \int \frac{\mathrm{d} \xi_{1}^{-} \mathrm{d} \xi_{2}^{-}}{(2 \pi)^{2}} e^{i \xi_{1}^{-} k_{1}^{+}+i \xi_{2}^{-} k_{2}^{+}} \\
& \times\left\{\left\langle p^{\prime}\right| g_{s} G^{+\rho}\left(\xi_{1}^{-} n\right) J^{+}\left(\xi_{2}^{-} n\right)\right. \\
& +\frac{i}{2} \Delta_{\perp v} G^{+\rho}\left(\xi_{1}^{-} n\right) G^{+\nu}\left(\xi_{2}^{-} n\right)|p\rangle \\
& +g_{\mu \nu} \int \mathrm{d} k_{3}^{+} \int \frac{\mathrm{d} \xi_{3}^{-}}{2 \pi} \mathrm{e}^{i k_{3}^{+}\left(\xi_{3}^{-}-\xi_{2}^{-}\right)} \\
& \left.\times\left\langle p^{\prime}\left|G^{+\rho}\left(\xi_{1}^{-} n\right) \tilde{D}_{\perp}^{\mu}\left(\xi_{3}^{-} n\right) G^{+v}\left(\xi_{2}^{-} n\right)\right| p\right\rangle\right\}
\end{aligned}
$$

It is noted that the matrix element with $G^{+-}$in Eq. (34) contains not only twist- 3 matrix element but also twist- 2 matrix element.

To the contribution from $\mathcal{M}_{1}^{\mu v}$ in Eq. (33), we have to add the factors

$\frac{k_{1}^{+}}{k_{1}^{+}-i \varepsilon}, \frac{k_{2}^{+}}{k_{2}^{+}-i \varepsilon}$,

in order to express the result with matrix elements of fieldstrength-tensor operators in the light-cone gauge $n \cdot G=0$. These factors can be different from 1 under integrations over $k_{1,2}^{+}$. We will discuss these added factors later. The contribution reads

$$
\begin{aligned}
\left.i \mathcal{T}\right|_{a, 32}= & g_{s}^{2} A_{J} \frac{16 m_{Q}^{2} \epsilon_{L}^{-}}{k^{+}\left(k^{-}\right)^{2}} \int \mathrm{d} k_{1}^{+} \mathrm{d} k_{2}^{+} \frac{\epsilon_{\rho}}{\left(k_{2}^{+}-i \varepsilon\right)^{2}\left(k_{1}^{+}-i \varepsilon\right)} \\
& \times \int \frac{\mathrm{d} \xi_{1}^{-} \mathrm{d} \xi_{2}^{-}}{(2 \pi)^{2}} e^{i \xi_{1}^{-} k_{1}^{+}+i \xi_{2}^{-} k_{2}^{+}} g_{\perp \mu \nu} \\
& \times\left\langle p^{\prime}\right|\left[k_{1}^{+} G^{+\mu}\left(\xi_{1}^{-} n\right) \tilde{\partial}_{\perp}^{\rho} G^{+\nu}\left(\xi_{2}^{-} n\right)\right. \\
& +\frac{i}{2} k_{1}^{+} \Delta_{\perp}^{\rho} G^{+\mu}\left(\xi_{1}^{-} n\right) G^{+\nu}\left(\xi_{2}^{-} n\right) \\
& -k^{+} G^{+\mu}\left(\xi_{1}^{-} n\right) \tilde{\partial}_{\perp}^{\nu} G^{+\rho}\left(\xi_{2}^{-} n\right) \\
& \left.-\frac{i}{2} k^{+} \Delta_{\perp \nu} G^{+\mu}\left(\xi_{1}^{-} n\right) G^{+\rho}\left(\xi_{2}^{-} n\right)\right]|p\rangle .
\end{aligned}
$$

This part contains not only twist-3 matrix elements but also those at twist- 2 matrix elements.

The contribution from Fig. 1b is relatively easy to obtain, because its leading contribution is at twist-3 in the light-cone gauge. We obtain

$$
\begin{aligned}
\left.i \mathcal{T}\right|_{b, 3}= & g_{s}^{3} A_{J} \frac{16 m_{Q}^{2} \epsilon_{L}^{*-}}{\left(k^{-}\right)^{2}} \int \mathrm{d} k_{1}^{+} \mathrm{d} k_{2}^{+} \mathrm{d} k_{3}^{+} \frac{-i^{3}}{\left(k_{2}^{+}-i \varepsilon\right)\left(k_{3}^{+}-i \varepsilon\right)} \\
& \times \frac{f^{a_{1} a_{2} a_{3}} g_{\perp \mu_{1} \mu_{2}} \epsilon_{\mu_{3}}}{\left(k_{2}^{+}+k_{3}^{+}-i \varepsilon\right)\left(k_{1}^{+}+k_{3}^{+}-i \varepsilon\right)} \\
& \times \int \frac{\mathrm{d} \xi_{1}^{-}}{(2 \pi)} \frac{\mathrm{d} \xi_{2}^{-}}{(2 \pi)} \frac{\mathrm{d} \xi_{3}^{-}}{(2 \pi)} e^{i \xi_{1}^{-} k_{1}^{+}+i \xi_{2}^{-} k_{2}^{+}+i \xi_{3}^{-} k_{3}^{+}} \\
& \left\langle p^{\prime}\left|G^{a_{1},+\mu_{1}}\left(\xi_{1}^{-} n\right) G^{a_{2},+\mu_{2}}\left(\xi_{2}^{-} n\right) G^{a_{3},+\mu_{3}}\left(\xi_{3}^{-} n\right)\right| p\right\rangle .
\end{aligned}
$$

Compared to the definitions in Eq. (6), this contribution can be expressed with the twist-3 matrix element $M_{F}^{\mu \nu \rho}$. It is noted that this contribution can also be expressed with $M_{D}^{\mu \nu \rho}$ by adding the contribution of the first term in the second line of Eq. (39). Since the three twist-3 matrix elements in Eq. (6) are not independent, we have the freedom to choose an independent set of these matrix elements. We will express our final results with $M_{D, \partial}^{\mu \nu \rho}$.

In deriving our results in the above, we have worked with the light-cone gauge $G^{+}=0$. In this gauge the gauge-field operator in the matrix elements can be converted to the fieldstrength-tensor operator combined with its momentum:

$$
\begin{aligned}
& \int r m d^{4} \xi_{i} \mathrm{e}^{i \xi_{i} \cdot k_{i}} k_{i}^{+} G^{\mu}\left(\xi_{i}\right) \\
& \rightarrow i \int r m d^{4} \xi_{i} \mathrm{e}^{i \xi_{i} \cdot k_{i}} G^{+\mu}\left(\xi_{i}\right), \quad i=1,2 .
\end{aligned}
$$

In our calculations the needed factors $k_{1,2}^{+}$are from $\mathcal{M}^{\mu \nu}$ and from the upper part of Fig. 1b, except for the contribution given in Eq. (39), where we have added the factors in Eq. (38). This introduces an ambiguity for the sign in front 
of $i \varepsilon$. It can be checked by doing the calculation in Feynman gauge. We have calculated the twist- 3 contributions of Fig. 1a in this gauge, and we found that the gauge fields in these contributions always appear in the combined form for $\mu \neq+$ :

$$
\int \mathrm{d}^{4} \xi_{i} e^{i \xi_{i} \cdot k_{i}}\left[G^{\mu}\left(\xi_{i}\right)-G^{+}\left(\xi_{i}\right) \frac{k_{\perp i}^{\mu}}{k_{i}^{+}-i \varepsilon}\right], \quad i=1,2 .
$$

This combination gives the form of field-strength-tensor operators at the leading order of $g_{s}$. This indicates that the sign of $i \varepsilon$ in Eq. (38) is correct. We have also calculated the contribution of Fig. 1b with one gluon field carrying the +-index in Feynman gauge. This contribution can be divided into two parts. One part gives the above combination; therefore, it is included in field-strength-tensor operators in Eq. (40) for Fig. 1b. If we take the contribution from Fig. 1a given in Eq. (39) as gauge invariant, which implies that there is the contribution from three-gluon exchanges with one gluon field carrying the +-index and the other two carrying a transverse index. This contribution comes partly from the non-linear term of the field-strength-tensor operators and partly from gauge links between operators. The remaining part from Fig. 1b with one gluon field carrying the + -index just gives this contribution. This gives an important check for gauge invariance of our results.

Our result for the amplitude at twist- 3 , denoted as $\mathcal{T}_{L}$, is the sum of the amplitudes given in Eqs. $(32,34,39,40)$. It is

$$
\begin{aligned}
i \mathcal{T}_{L}= & 2 g_{s}^{2} A_{J} \frac{\epsilon_{\rho}}{m_{Q}}\left\{\int \mathrm{d} x \frac{1}{(x+\xi-i \varepsilon)^{2}(\xi-x-i \varepsilon)}\right. \\
& {\left[i \Delta_{\perp}^{\rho}\left(\xi-x-\rho_{L}(x+\xi)\right) \mathcal{F}_{g}(x, \xi, t)\right.} \\
& \left.+2 \xi \epsilon_{\perp}^{\mu \rho} \Delta_{\mu} \tilde{\mathcal{F}}_{g}(x, \xi, t)\right] \\
& +4 \xi \int \mathrm{d} x_{1} \mathrm{~d} x_{2}\left[\frac{\delta\left(x_{1}-x_{2}\right) \tilde{G}_{\partial}^{\rho}\left(-x_{1}, \Delta\right)}{\left(x_{1}+\xi-i \varepsilon\right)^{2}\left(\xi-x_{1}-i \varepsilon\right)}\right. \\
& +\frac{\tilde{G}_{D}^{\rho}\left(x_{1}, x_{2}, \Delta\right)}{\left(x_{2}+\xi-i \varepsilon\right)^{2}\left(\xi-x_{2}-i \varepsilon\right)} \\
& +\frac{G_{D}^{\rho}\left(x_{1}, x_{2}, \Delta\right)}{\left(\xi+x_{1}-i \varepsilon\right)\left(\xi-x_{1}-i \varepsilon\right)\left(\xi+x_{2}-i \varepsilon\right)} \\
& \left.\left.\left.+\frac{\sum_{q} N_{F}^{\rho}\left(x_{1}, x_{2}, \Delta\right)}{\left(2 \xi-x_{2}+x_{1}-i \varepsilon\right)^{2}\left(x_{2}-x_{1}-i \varepsilon\right)}\right)\right]\right\} .
\end{aligned}
$$

This result is complicated when we express it with various GPDs. Totally, 20 GPDs or scalar functions are involved with the parameterization of twist- 2 and twist- 3 matrix elements given before. This makes the extraction of GPDs from experiment complicated. Unless one can use some nonperturbative methods to calculated these GPDs, it seem impossible to make reliable predictions for experiment.

Before giving a discussion about our results, we notice that our amplitudes in Eqs. $(29,43)$ are $U_{e m}(1)$-gauge invariant only at the leading order of $\lambda$. Since in our results only the transverse part of the polarization vector of the initial photon is involved, the amplitudes are not exactly zero by replacing the polarization vector with the photon momentum $q^{\mu}$. After replacement the amplitudes are in fact suppressed by one power of $\lambda$ because $q_{\perp}^{\mu}$ is at order of $\lambda$. Hence, the $U_{e m}(1)$ gauge invariance of our result holds only at the leading order of $\lambda$.

One may expect that the amplitude can be simplified in the high energy limit, i.e., $s \gg m_{Q}^{2}$. In the process $\xi$ is fixed as $\xi=2 m_{Q}^{2} /\left(s-2 m_{Q}^{2}\right)(1+\mathcal{O}(\lambda)$. Formally, one sets $\xi=0$ to significantly simplify the twist-3 amplitude in the limit $s \gg m_{Q}^{2}$. But this will result in a divergent contribution. This can be seen from the contribution from the GPD $H_{g}(x, \xi, t)$ contained in $\mathcal{F}_{g}$. In the limit $\Delta^{\mu} \rightarrow 0 H_{g}$ is related to the gluon parton distribution $g(x)$ as $H_{g}(x, 0,0)=x g(x)$. For $x \rightarrow 0 g(x)$ behaves as $x^{-\alpha}$ with $\alpha>0$. The pole contribution from $H_{g}$ in the first line of Eq. (43) is expected for $\xi \rightarrow 0$ as $\xi^{-\alpha}$, as discussed in [31]. The behavior of GPDs in the region $|x| \sim \xi$ is crucial for the limit. It is clear that the integration regions with $|x| \sim \xi$ and $\left|x_{1,2}\right| \sim \xi$ are the most important ones. If one assumes that all GPDs have similar behaviors in these regions, one may only neglect the last two terms by power counting of $\xi$. Thus, the behaviors of the GPDs may have a significant effect on our calculation. One behavior of the GPDs has been studied in a series of papers [16-23], which show that some twist-3 GDPs may be discontinuous in some region. If this is true for the twist-3 GPDs in Eq. (43), we will not be allowed to do some of the integrations there. Thus, the power counting method cannot be applied to each twist-3 term of Eq. (43) individually. However, one has also shown [16] that some particular linear combinations of the twist-3 GPDs are free of discontinuity, and the amplitude may only depend on these linear combinations, although these GPDs may be discontinuous individually. The verification of a similar conclusion as regards the twist-3 GPDs here is another long work which is out of scope of this paper. Before this verification, one should always be careful when making use of the results here directly. Additionally, if a similar conclusion should be drawn here, it may be helpful to explain the poles of the hard factors which are convoluted to the twist-3 GPDs in Eq. (43). In general, it is not clear if the amplitude will be simplified in the limit. But the relative order of importance between twist-2- and twist-3 amplitude can be estimated, if we assume that the contributions from twist-3 GPDs can be transformed into terms that are free of discontinuity and, therefore, are finite.

By simply comparing the contribution from $H_{g}$ in twist2- or twist-3 contributions, one can already conclude that the twist- 3 amplitude is suppressed only by a factor $\Delta_{\perp} / m_{Q}$. For $J / \psi m_{Q}$ is $m_{c}$; around $1 \mathrm{GeV}$. Therefore, the suppression is not strong. The weak suppression has a significant impact on 
extracting twist-2 gluon GPDs by using the leading amplitude given in Eq. (29), in which $J / \psi$ is only transversely polarized. If the polarization of $J / \psi$ is not measured or summed in experiment, then there will be a substantial contribution of longitudinally polarized $J / \psi$ in the measured differential cross-sections. This can result in the extracted twist-2 GPDs by using the leading amplitude being not accurate and containing significant effects of higher-twists. This is an important observation of our work.

From the production of transversely polarized $J / \psi$ or $\Upsilon$ one can only extract the gluon GPDs $E_{g}$ and $H_{g}$. It is possible to obtain information of other twist-2 GPDs through production of quarkonium with different quantum numbers. At leading power or twist-2 level, the exchanged two gluons in Fig. 1a can only be in a state with the quantum number $C=+$. Therefore, $h_{c}$ can be produced at twist-2. Other quarkonia, like $\eta_{c}$ and $\chi_{c J}$, with $J=0,1,2$, can only be produced through three-gluon exchange given in Fig. 1b, where the production is described with twist-3 GPDs obtained from $M_{F}^{\mu \nu \rho}$ by replacing if $a b c$ in Eq. (6) by $d^{a b c}$.

\section{Calculations for $h_{c}$ Production}

We have derived the twist-2- and twist-3 amplitude of $h_{c}$. At twist- $2 h_{c}$ is transversely polarized. The amplitude reads

$$
\begin{aligned}
\mathcal{T}_{T}= & 16 g_{s}^{2} A_{P} \epsilon_{v}^{*}(k) \epsilon^{\rho}(q) \epsilon_{\perp \mu \rho} \\
& \times \int \mathrm{d} x \frac{\xi}{(\xi-x-i \varepsilon)^{2}(\xi+x-i \varepsilon)} \\
& \times\left[g_{\perp}^{\mu \nu} \mathcal{F}_{g}-i \epsilon_{\perp}^{\mu \nu} \tilde{\mathcal{F}}_{g}+2 \mathcal{F}_{T g}^{\mu \nu}\right], \\
A_{P}= & e \sqrt{\frac{3}{4 \pi N_{c}}} R^{\prime *}(0)\left(2 m_{Q}\right)^{-5 / 2},
\end{aligned}
$$

where $R^{\prime}(0)$ is the derivative of the radial wave function at the origin. It is interesting to note that all twist- 2 gluon GPDs are involved. But the production of $h_{c}$ can be difficult to measure. At twist- $3 h_{c}$ is longitudinally polarized. The amplitude is

$$
\begin{aligned}
\mathcal{T}_{L}= & \frac{4 g_{S}^{2} A_{P}}{m_{Q}} \int \mathrm{d} x\left\{\tilde{\epsilon} \times \Delta_{\perp} \mathcal{F}_{g}\left[\frac{-2}{(\xi-x-i \varepsilon)(\xi+x-i \varepsilon)}\right.\right. \\
& +\xi\left(\frac{5 x-3 \xi}{(\xi-x-i \varepsilon)(\xi+x-i \varepsilon)^{3}}\right. \\
& \left.\left.+\frac{\rho_{L}}{(\xi-x-i \varepsilon)^{2}(\xi+x-i \varepsilon)}\right)\right] \\
& +i \xi \epsilon \times \Delta_{\perp} \tilde{\mathcal{F}}_{g}\left[\frac{2 x-6 \xi}{(\xi-x-i \varepsilon)(\xi+x-i \varepsilon)^{3}}\right. \\
& \left.-\frac{\rho_{L}}{(\xi-x-i \varepsilon)^{2}(\xi+x-i \varepsilon)}\right]
\end{aligned}
$$

$$
\begin{aligned}
& -\xi F_{T}^{\mu \nu}\left[\frac{(10 \xi-6 x) \tilde{\epsilon}_{\mu} \Delta_{\perp v}}{(\xi-x-i \varepsilon)(\xi+x-i \varepsilon)^{3}}\right. \\
& \left.\left.+\frac{\rho_{L} \epsilon_{\mu} \tilde{\Delta}_{\perp v}}{(\xi-x-i \varepsilon)^{2}(\xi+x-i \varepsilon)}\right]\right\} \\
& +\frac{8 g_{s}^{2} A_{P} \xi}{m_{Q}} \int \mathrm{d} x_{1} \mathrm{~d} x_{2}\left\{-\frac{4 \tilde{\epsilon}_{\mu} \tilde{G}_{D}^{\mu}\left(x_{1}, x_{2}\right)}{\left(\xi+x_{2}-i \varepsilon\right)^{3}}\right. \\
& +2 \tilde{\epsilon}_{\mu} G_{\partial}^{\mu}\left(x_{1}\right) \delta\left(x_{1}-x_{2}\right) \frac{\xi-2 x_{1}}{\left(\xi-x_{1}-i \varepsilon\right)^{2}\left(\xi+x_{2}-i \varepsilon\right)^{2}} \\
& +\frac{\left(x_{1}+x_{2}\right) \tilde{\epsilon}_{\mu} G_{D}^{\mu}\left(x_{1}, x_{2}\right)}{\left(\xi-x_{2}-i \varepsilon\right)\left(\xi+x_{1}-i \varepsilon\right)\left(\xi-x_{1}-i \varepsilon\right)\left(\xi+x_{2}-i \varepsilon\right)} \\
& +4 \tilde{\epsilon}_{\mu} \tilde{G}_{\partial}^{\mu}\left(x_{1}\right) \delta\left(x_{1}-x_{2}\right) \frac{x_{1}}{\left(\xi-x_{1}-i \varepsilon\right)^{2}\left(\xi+x_{1}-i \varepsilon\right)^{2}} \\
& \left.-4 \tilde{\epsilon}_{\mu} \frac{\sum_{q} N_{F}^{\mu}\left(x_{1}, x_{2}\right)}{\left(2 \xi+x_{1}-x_{2}-i \varepsilon\right)^{3}}\right\},
\end{aligned}
$$

with $\tilde{\epsilon}^{\mu}=\epsilon_{\perp}^{\mu \nu} \epsilon_{v}$ and $\tilde{\Delta}^{\mu}=\epsilon_{\perp}^{\mu \nu} \Delta_{v}$. Again, the amplitude here is suppressed only by the inverse of $m_{Q}$ in comparison with the twist-2 amplitude in Eq. (44). But this amplitude is in fact divergent.

Although GPDs cannot be calculated perturbatively, their dependence on the renormalization scale $\mu$ can be calculated with perturbative theory if $\mu$ is large enough. The evolutions of twist-2 GPDs have been studied in [36]. The asymptotic behaviors have been derived. In the limit of $\mu \rightarrow \infty$ the $x$ dependence of the GPD $E_{g}$ and $H_{g}$ contained in $\mathcal{F}_{g}$ can be found in $[8,36]$ :

$$
\begin{aligned}
& E_{g}(x, \xi) \sim \frac{4 C_{F}}{4 C_{F}+n_{f}} \frac{1}{\xi^{5}}(\xi-x)^{2}(\xi+x)^{2} \theta(\xi-|x|), \\
& H_{g}(x, \xi) \sim \frac{4 C_{F}}{4 C_{F}+n_{f}} \frac{1}{\xi^{5}}(\xi-x)^{2}(\xi+x)^{2} \theta(\xi-|x|) .
\end{aligned}
$$

In the limit these two GPDs are zero for $|x|>\xi$. Given such a dependence, the integrals like

$$
\int \mathrm{d} x \frac{1}{(\xi+x-i \varepsilon)^{3}}\left\{E_{g}(x, \xi), H_{g}(x, \xi)\right\}
$$

are divergent in the limit $\mu \rightarrow \infty$. The last term in the first line in Eq. (45) contains such integrals but at finite $\mu$. One can calculate these integrals at $\mu \rightarrow \infty$ and obtain the results at finite $\mu$ from the evolution of the two GPDs. The evolutions cannot eliminate such divergences. Therefore, the results in Eq. (45) are in fact divergent. The divergences come from the momentum region where one of the two exchanged gluons in Fig. 1a is soft. Such divergences are end-point ones, which are well known in collinear factorization of exclusive processes with light-cone amplitudes of hadrons, e.g., in twist- 3 contributions of the $\pi$ form factor and exclusive $B$-meson decays. For processes factorized with GPDs, the existence of such divergences involving GPDs has been first observed in $\rho$-meson production in [31]. These divergences make QCD factorization impossible [32-35]. To the best of 
our knowledge, there has so far appeared no rigorous way to deal with the singularities.

It should be emphasized that the existence of the endpoint singularity in our twist-3 amplitude of $h_{c}$ only means that we cannot evaluate it correctly in the framework of collinear factorization. But the contribution of the amplitude is power-suppressed. The amplitude of $h_{c, b}$ at leading power or twist-2 has no such a singularity. If the power correction can be neglected, a physical prediction can still be made from Eq. (44) for $h_{c, b}$ production. It is noted that in the twist3 amplitude of $J / \psi$ or $\Upsilon$ in Eq. (43) there is no evidence of existing end-point singularities. One can expect that the factorization holds in this case.

\section{Summary}

To summarize, we have studied at twist-3 level the forward photoproduction of a quarkonium, where the quarkonium can be $J / \psi$ and $h_{c}$. We have classified the relevant twist3 gluon GPDs and studied their properties. The produced quarkonium at twist-2 is transversely polarized. At twist-3 it is longitudinally polarized and the amplitude is powersuppressed. Our result indicates that the twist-3 amplitude of $h_{c}$ contains end-point singularities which spoil the factorization. We find that the twist-3 amplitude is only suppressed by the inverse of the heavy quark mass. This has an important implication for extracting twist-2 gluon GPDs, if the polarization of the produced quarkonium is not observed. In this case, the contribution of the longitudinal polarization can be significant and should be taken into account. Experimentally, the studied processes can be already studied at JLab, and in the future at EIC or After@LHC as proposed in [37] or [38], respectively.

Acknowledgements The work is supported by National Nature Science Foundation of P.R. China (No. 11675241 and No. 11821505). The partial support from the CAS center for excellence in particle physics(CCEPP) is acknowledged.

Data Availability Statement This manuscript has no associated data or the data will not be deposited. [Authors' comment: In this paper, we do not use any data.]

Open Access This article is distributed under the terms of the Creative Commons Attribution 4.0 International License (http://creativecomm ons.org/licenses/by/4.0/), which permits unrestricted use, distribution, and reproduction in any medium, provided you give appropriate credit to the original author(s) and the source, provide a link to the Creative Commons license, and indicate if changes were made.

Funded by SCOAP ${ }^{3}$.

\section{References}

1. D. Mueller et al., Fortsch. Phys. 42, 101 (1994)

2. A.V. Radyushkin, Phys. Lett. B 380, 417 (1996) arXiv:hep-ph/9604317

3. A.V. Radyushkin, Phys. Lett. B 385, 333 (1996). arXiv:hep-ph/9605431

4. X. Ji, Phys. Rev. Lett. 78, 610 (1997). arXiv:hep-ph/9603249

5. X. Ji, Phys. Rev. D 55, 7114 (1997). arXiv:hep-ph/9609381

6. D. Mueller, Few Body Syst. 55, 317 (2014). arXiv:1404.2817 [hep$\mathrm{ph}]$

7. M. Diehl, Phys. Rept. 388, 41 (2003). arXiv:hep-ph/0307382

8. A.V. Belitsky, A.V. Radyushkin, Phys. Rept. 418, 1 (2005). arXiv:hep-ph/0504030

9. X. Ji, X. Xiong, F. Yuan, Phys. Rev. Lett. 109, 152005 (2012). arXiv:1202.2847 [hep-ph]

10. X. Ji, X. Xiong, F. Yuan, Phys. Rev. D88, 014041 (2013). arXiv:1207.5221 [hep-ph]

11. Y. Hatta, Phys. Lett. B708, 186 (2012). arXiv:1111.3547 [hep-ph]

12. Y. Hatta, S. Yoshida, JHEP 1210, 080 (2012). arXiv:1207.5332 [hep-ph]

13. DYu. Ivanov, A. Schafer, L. Szymanowski, G. Krasnikov, Eur. Phys. J. C 34, 297 (2004). arXiv:hep-ph/0401131

14. M. Vanttinen, L. Mankiewicz, Phys. Lett. B 440, 157 (1998). arXiv:hep-ph/9807287

15. J. Koempel, P. Kroll, A. Metz, J. Zhou, Phys. Rev. D 85, 051502 (2012). arXiv:1112.1334

16. Fatma Aslan, Matthias Burkardt, Phys. Rev. D 98, 014038 (2018)

17. A.V. Belitsky, D. Mueller, Nucl. Phys. B 589, 611 (2000)

18. N. Kivel, M.V. Polyakov, A. Schäfer, O.V. Teryaev, Phys. Lett. B 497, 73 (2001)

19. A.V. Radyushkin, C. Weiss, Phys. Lett. B 493, 332 (2000)

20. N. Kivel, M.V. Polyakov, Nucl. Phys. B 600, 334 (2001)

21. A.V. Radyushkin, C. Weiss, Phys. Rev. D 63, 114012 (2001)

22. A.V. Belitsky, D. Mueller, A. Kirchner, A. Schäfer, Phys. Rev. D 64, 116002 (2001)

23. N. Kivel, M.V. Polyakov, M. Vanderhaeghen, Phys. Rev. D 63, 114014 (2001)

24. G.T. Bodwin, E. Braaten and G. Peter Lepage, Phys.Rev. D51 1125, (1995) Erratum-ibid.D55 (1997) 5853, e-Print arXiv:hep-ph/9407339

25. A.V. Efremov, O.V. Teryaev, Sov. J. Nucl. Phys 36, 142 (1982)

26. A.V. Efremov, O.V. Teryaev, Phys. Lett B150, 383 (1985)

27. J.W. Qiu, G. Sterman, Phys. Rev. Lett 67, 2264 (1991)

28. J.W. Qiu, G. Sterman, Nucl. Phys. B 378, 52 (1992)

29. J.W. Qiu, G. Sterman, Phys. Rev D59, 014004 (1998)

30. R.L. Jaffe, A. Manohar, Nucl. Phys. B 337, 509 (1990)

31. L. Mankiewicz, G. Piller, Phys. Rev. D 61, 074013 (2000). arXiv:hep-ph/9905287

32. J. Collins, L. Frankfurt, M. Strikman, Phys. Rev. D 56, 2982 (1997)

33. A. Radyushkin, Phys. Lett. B 380, 417 (1996)

34. A. Radyushkin, Phys. Lett. B 385, 333 (1996)

35. A. Radyushkin, Phys. Rev. D 56, 5524 (1997)

36. A.V. Radyushkin, Phys. Rev. D 59, 014030 (1999). arXiv:hep-ph/9805342

37. A. Accardi et al., Eur. Phys. J. A52(9), 268 (2016). arXiv:1212.1701 [nucl-ex]

38. L. Massacrier, J.P. Lansberg, L. Szymanowski, J. Wagner, arXiv: 1709.09044 [nucl-ex] 